\title{
The Secret to Successful User Communities: \\ An Analysis of Computer Associates' User Groups
}

Celine Schulz

Discussion paper 2006-09

November 2006

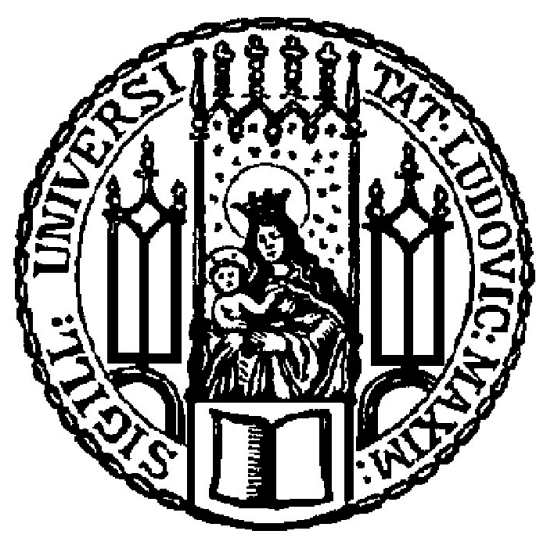

Munich School of Management

University of Munich

Fakultät für Betriebswirtschaft

Ludwig-Maximilians-Universität München

Online at http://epub.ub.uni-muenchen.de/ 


\title{
The Secret to Successful User Communities: An Analysis of Computer Associates' User Groups
}

\author{
Celine Schulz* \\ University of Munich
}

31, July 2006

\begin{abstract}
This paper provides the first large scale study that examines the impact of both individual- and group-specific factors on the benefits users obtain from their user communities. By empirically analysing 924 survey responses from individuals in 161 Computer Associates' user groups, this paper aims to identify the determinants of successful user communities. To measure success, the amount of time individual members save through having access to their user networks is used. As firms can significantly profit from successful user communities, this study proposes four key implications of the empirical results for the management of user communities.
\end{abstract}

Keywords: Managing User Communities, Collective Action, Information Sharing

Acknowledgements: I am indebted to Karin Hoisl, Carolin Haeussler, Georg von Graevenitz and Philip Mayrhofer for their valuable comments and discussions. Thank you to the many user group presidents and members who gave graciously and thoughtfully of their time and experience. Financial support from the Volkswagen Stiftung is gratefully acknowledged.

*Institute for Innovation Research, Technology Management and Entrepreneurship, Munich School of Management, Kaulbachstraße 45, D-80539, Munich, Tel: +49 892180 3760, e-mail: cschulz@ bwl.uni-muenchen.de 


\section{Introduction}

Groups of customers in the form of user communities can exercise a powerful influence on the market, due to the speed with which they can disseminate information (Prahalad and Ramaswamy 2000). For instance, closely-knit, networked groups of customers can play a vital role for the adoption and diffusion of innovations (Chakravorti 2004). Furthermore, user communities can also be seen as an additional source of external ideas for firms pursuing an open innovation model (Chesbrough 2003). In particular, these communities have the potential to assist firms in organising and prioritising the pool of user information regarding their needs, feedback to existing products as well as ideas for new products. The access to and integration of such community-generated information have been demonstrated by various studies to contribute significantly to product development (Jeppesen and Molin 2003; Jeppesen and Frederiksen 2006; Prügl and Schreier 2006). But before firms can leverage user communities for innovation management, it is important to firstly understand why these user communities exist, what their goals are and how they function. To address these issues, this paper examines user groups from a proprietary software producer Computer Associates (CA), and aims to identify determinants of successful user communities.

An important premise for the existence of user communities is that the transmission and acquisition of (tacit and codified) knowledge can be both difficult and costly. In contrast to codified knowledge, tacit knowledge can be neither expressed in a particular language nor recorded on a particular medium, and is as a result not readily transferable (Cowan et al. 2000; Foray 2004). The term "tacit" was first introduced by Polyani (1966), who proposed the notion that individuals can know more than they can tell, because they are often not conscious of the knowledge they possess. Frequent interactions with their user communities can assist users to exchange both tacit and codified knowledge. This is because these regular exchanges foster the establishment of common cognitive platforms and common social norms (Cohendet 2006), which in turn increase the willingness of individuals to share such knowledge with each other. Furthermore, the easy access to potential knowledge pools within user communities can significantly reduce costs users incur when searching for solutions to their problems; or for innovative ideas as to how they can better optimise the use of their products (Lakhani and von Hippel|2003; Franke and Shah 2003).

To understand how user communities function, the theory of collective action (Olson 1965) is used to examine the incentives and mechanisms that motivate individuals to act in support of their group interests. With the phenomenal success of open source software in the last decade, interest in the theory of collective action and its application in explaining the microfoundations of open source communities soared (von Hippel and von Krogh 2003; von Krogh et al. 2003a). The reasons as to why these horizontal innovation networks are successful, have been widely discussed von Hippel 2002). Apart from having analysed the extrinsic and intrinsic motivations - such as future 
career benefits, skill improvement, fun, reciprocity and altruism - of individual programmers to voluntarily contribute to a public good (Lerner and Tirole 2002; Lakhani and Wolf 2005), studies have also examined the organisational structures responsible for the internal functioning of these communities (Weber 2004).

The objective of this study is to analyse the impact of both individual- and group-specific factors on the benefits users receive from their user communities. As rational individuals will only participate in and contribute to their user community if they perceive a net benefit, this knowledge is of particular importance for the management of successful user communities. This paper hence contributes to the literature on the management of user communities by providing a discussion of how user communities should be organised such that members' benefits are maximised. To quantify user benefits, the amount of time individual members save through having access to their user communities is used. The empirical analysis is based on 924 survey responses from individuals in 161 Computer Associates' user groups.

Hypotheses for the individual- and group-specific factors are derived from a diversity of disciplines ranging from sociology and psychology to economics and business. The empirical results propose that the following determinants have an impact on the benefits individuals are able to receive from their user communities: the number of intra-group informal ties, the amount of time users invest in their groups, the communication culture of the group, the number of star members in the group and the groups' membership requirements.

In Section 2 of this paper, extant relevant literature will be reviewed. Next, the hypotheses of the determinants of successful user communities will be derived in Section 3. This will be followed by the research methodology in Section 4 and a short introduction to Computer Associates and its user groups in Section 5. Section 6 describes the variables to be used in the empirical analysis and Section 7 presents the descriptive statistics, the results of the multivariate analysis, and a discussion of the implications of these findings for the management of user communities. Section 8 concludes.

\section{Literature Review}

Olson (1965) was the first scholar to formalise the notion of collective action and the free rider problem ${ }^{1}$ in his book, The Logic of Collective Action. According to Olson, the characteristic and primary function of organizations is to advance the common interests of groups of individuals.

\footnotetext{
${ }^{1}$ Modern political theorists in the eighteenth century such as David Hume, Alexander Hamilton, James Madison, and John Stuart Mill had developed theories of the free riding behaviour way before Olson's work. However, most of these theories were hidden in written works that were totally disregarded in the mid-twentieth century until Olson gave them relevance again (Dougherty 2003$)$.
} 
Hence, it can be considered that when any common interest of the group has been satisfied, then a collective good has been provided for that group. Olson particularly addressed the issue of the conflict between individual and collective interests and contended that in the absence of coercion and some separate individual incentive, rational self-interested individuals in a group will seek to maximise their personal welfare and not act to advance the interest of the group to which they belong to. His central thesis was that larger groups are less likely to succeed in achieving their goals as compared to smaller groups.

Olson supported his thesis with the argument that individuals will tend to free ride ${ }^{2}$ on the contributions of others in large groups. This is because an increase in the size of the group decreases the likelihood that the contribution and non-contribution of any one individual will be perceptible. In other words, if one member does or does not help to provide the collective good, no other one member will be significantly affected. Hence, individuals have little or no incentive to contribute to the group. When this free riding behaviour is in the interest of most or all members in the group, there would be an under-provision or even a non-provision of the collective good - this outcome is often termed the free rider problem.

Since the successful functioning of any organisation must be based on some circumvention of this free rider problem (Frohlich and Oppenheimer 1970), a great deal of research on the issue of collective action in the last half century has focused on various incentives and mechanisms that mitigate this problem. In the following, these incentives and mechanisms will be illustrated using examples from the conventional collective action literature and the more recent open source software literature.

Although Olson (1965) first introduced the idea that the presence of separate incentives can motivate individuals to advance the interest of the group they belong to, it was Hardin (1982) who focused his work on the internal incentives that motivate groups to act. Separate incentives can be considered extrinsic motivations, which refer to the separable outcome (or indirect reward) that is attained when an activity is done (Ryan and Deci 2000). One such separate incentive Hardin (1982) addressed, was the political entrepreneurship motivation, which occurs when individuals, who for their own career reasons, find it in their private interest to work to provide collective benefits to relevant groups. Lerner and Tirole (2001, 2002) propose a similar separate incentive, which they refer to as the signaling motive for career advancements and/or future career benefits. Additional extrinsic motivations from the open source software literature include: monetary rewards, skill improvement and active peer review (Ghosh et al. 2002; Hertel et al. 2003; Lakhani and Wolf

\footnotetext{
${ }^{2}$ Free riding can also be defined as the failure of individuals to reveal their true preferences for a collective good through their contributions (Cornes and Sandler 1996). Free riders are hence individuals who see incentives to withhold their resources, hoping that the efforts of others will suffice to provide the good (Frohlich and Oppenheimer 1970).
} 
2005).

Besides extrinsic motivations, Hardin (1982) also acknowledged the existence of intrinsic motivations, which refer to the inherent satisfaction of the doing of an activity (Ryan and Deci 2000). Hardin's example of an intrinsic motivation was his concept of extrarational motivations, which occur when the unselfish and social motives of members bring about the provision of a collective good. For example, fervent nationalism might inspire a group of individuals to organise a political party. The social norm, reciprocity is also an example of an intrinsic motivation. Reciprocity refers to the behaviour of individuals to react to positive actions of others with positive responses and to negative actions of others with negative responses (Ostrom 1998). Hence individuals may be willing to contribute to the group because they have benefited from the contributions of other group members in the past. Additional intrinsic motivations from the open source software literature include: fun (Torvalds and Diamond 2001; Lakhani and Wolf 2005; Bitzer et al. 2004), reputation (Ghosh et al.2002; Lakhani and Wolf 2005), and altruism (Zeitlyn 2003; Bitzer et al. 2004).

Without the presence of a formal organisation or an informal group agreement, individuals may have insufficient intrinsic and extrinsic motivations to voluntarily contribute to the group (Olson 1965; Hardin 1982). Hence, it is sometimes necessary for a central authority, in the form of an external institution or a group leader, to establish control mechanisms in the group that encourage members to participate and discourage free-riding (von Hippel and von Krogh 2003). Examples of such control mechanisms include: facilitating the coordination between members, monitoring their contributions, or enforcing penalties against free riding (Oliver 1980; Swanson 1992; Taylor and Singleton 1993). Additionally, the central authority can also promote a collective identity (Friedman and McAdam 1992) and social norms such as reciprocity, reputation and trust (Ostrom 1998) between members in the group. As mentioned above, such norms can be referred to as intrinsic motivations that can help solve social dilemmas ${ }^{3}$ such as the free rider problem (Ostrom 1998).

In the following, the importance of group leadership to coordinate and monitor members' contributions of two successful open source software projects are presented. It is a common misconception that open source communities are simply "networked hordes of programmer/anarchists" (Raymond 1999). Although individuals are intrinsically and extrinsically motivated to contribute to the open source community, it is nevertheless necessary for an individual or a group of individuals to take the lead in coordinating and organising community activities (Weber 2004). One of the most successful open source software, Linux was created in 1991 by Linus Torvalds. In its

\footnotetext{
${ }^{3}$ Social dilemmas are situations when individuals face choices in which the maximisation of short-term self-interest yields outcomes that leave all participants worse off than feasible alternatives (Ostrom 1998). In other words, each individual will receive a higher payoff for defecting than for cooperating, but all are better off if all cooperate than if all defect.
} 
early development stages, the Linux project was chiefly led and controlled by Torvalds. As the size of the community of developers increased, Torvalds reorganised his community and delegated responsibilities for subsystems and components of the kernel to a core group of developers. As a result of his high involvement and control of the Linux project, Torvalds is also sometimes known as its "Benevolent Dictator" (Torvalds and Diamond 2001; Weber 2004). In contrast to Linux, the Apache server software project did not originate from a single leader, but rather a core group of seven developers in 1994 (von Hippel 2001; Lerner and Tirole 2002). The group devised a voting system based on a minimal quorum consensus rule to collectively involve all developers who contributed to Apache in the group's decision-making processes (Mockus et al. 2000). This voting system is used not only to make code changes but also for the inclusion of new members (Fielding 1999).

To summarise, the theoretical and empirical literature on the conventional theory of collective action and open source software suggest that well managed groups can assist in promoting the intrinsic and/or extrinsic motivations of individuals to participate in and contribute to the group to which they belong to.

\section{Determinants of Successful User Communities}

This study will base its analysis on a specific type of organisation of individuals, that of user communities. User communities are defined as groups of individuals who all use a similar product and who learn how to use it better as they interact on a regular basis 4 (Wenger 2004). Through the sharing of ideas, insights and experiences with each other, user communities provide benefits for their members. For instance, access to the user network and community documents can save individuals valuable time (McDermott 2002; Lakhani and von Hippel 2003). This paper hence defines successful user communities as groups whose members have benefited significantly from their acquisition of knowledge and information from their user community. To quantify users' benefits, the amount of time individual members save through having access to their user networks is used. In the following, hypotheses of the impact of individual- and group-specific factors on users' benefits will be derived.

\subsection{Individual-Specific Determinants}

\section{Number of Strong Ties}

A tie is a relationship between two individuals. The strength of a tie is the combination of the

\footnotetext{
${ }^{4}$ Franke and Shah $(2003)$ used a similar 'communities of practice' definition for their notion of user communities.
} 
amount of time, the emotional intensity, the intimacy (mutual confiding), and the reciprocal services which characterise the tie (Granovetter 1973). These relational ties between individuals can be considered channels for the transfer or the flow of resources, such as information and knowledge (Wasserman and Faust 1994).

Although Granovetter (1973, 1983) proposes that weak ties distribute information more efficiently because it provides individuals with access to information and resources beyond those available in their own social circles, he acknowledges that strong ties have greater motivation to be of assistance and are generally more easily available. Applying Granovetter's strong tie hypothesis to the study of entrepreneurship, Jack (2005) finds that strong ties are instrumental for business activity and are used extensively to provide knowledge and information, as well as to maintain, extend and enhance business and personal reputations. Carpenter et al. (2003) also find that in inter-organisational contact-making in U.S. health politics, individuals tend to convey new information to their strong ties.

As individuals with a greater number of close contacts may be more accessible to receive new information and potential assistance to difficulties they encounter, it is expected that

H1: Users who have a larger number of strong ties to other members benefit more from their user communities.

\section{Learning Ability}

An individual's experience on a series of related or similar tasks may improve his ability to learn new tasks. This progressive improvement in the performance of learning tasks is a form of knowledge transfer known as learning to learn (Ellis 1965; Estes 1970). To illustrate this, Ward (1937) conducted an experiment where participants were required to learn successive lists of twelve syllables, one list a day, which were all about equal in difficulty. He found that participants required approximately thirty-eight trials to completely master the first list; twenty trials to reach mastery after six lists; and only fourteen trials to reach mastery after fifteen lists. Improvement in participants' learning rates were most rapid during the first six lists and were more gradual afterwards. Similar results were obtained in a study by Melton and von Lackum (1941).

As the investment in an individual's learning ability is expected to positively impact his ability to identify, assimilate and use new knowledge (Cohen and Levinthal 1990), it can be assumed that:

H2: Users who invest more time in improving their learning abilities benefit more from their user communities. 


\section{Face-to-Face Communication}

It has been shown that face-to-face communication promotes higher levels of cooperation $\sqrt[5]{(\text { Ostrom }}$ 1998; Ostrom et al. 1994). In particular, Sally (1995) finds that when participants communicate face-to-face in one-shot prisoners' dilemma and public good experimental games, their cooperation rate rises, on average, by more than 45 percentage points.

Additionally, face-to-face settings help promote the transfer of tacit knowledge. As tacit knowledge can be neither expressed in a particular language nor recorded on a particular medium (Cowan et al. 2000; Foray 2004), face-to-face dialogues or interactions can facilitate the articulation of tacit knowledge into codified knowledge (von Krogh 1998; Foray 2004). Polyani (1958) contends that such tacit knowledge can only be passed on by example from master to apprentice. Hence,

H3: Users benefit more from face-to-face interactions as compared to other forms of communication with their user communities.

\subsection{Group-Specific Determinants}

\section{Open Communication}

An open exchange of information within groups can limit conflict between members (Lau and Murninghan 1998). Furthermore, a culture that encourages a free flow of knowledge would encourage individuals to share more of their knowledge with each other (von Krogh 1998), thus increasing the quantity of the potential knowledge pool in the community.

Shah (2005) finds that open communication amongst users in innovation communities can increase the diversity of expertise in problem solving and allow the results of trial-and-error experimentation by multiple parties to be exchanged. Furthermore, open communication not only increases the likelihood that users find effective solutions to their problems, but also reduces the time required to find or create such solutions.

Therefore, the following relationship is expected:

H4: Users benefit more from user communities that have an open culture of communication.

\section{Number of Star Members}

Star members in open source software projects refer to the core group of developers who contribute most of the code and oversee the design and evolution of the project (Crowston et al. 2006, von

\footnotetext{
${ }^{5}$ See Ostrom (1998) for an overview of experimental studies that find a positive effect of face-to-face communication on cooperation.
} 
Krogh et al.2003b). In the case of the biotechnology industry, Zucker and Darby (1996) found that the number of collaborative links to star scientists had significant positive effects on the success ${ }^{6}$ of new biotechnology enterprises. These star scientists were highly productive researchers who accounted for only $0.8 \%$ of all scientists listed in GenBank 7 through to 1990 , but for $17.3 \%$ of all published articles.

Similar to open source software communities, star members in user communities are characterised as having a high level of experience, skill and knowledge of the product they use. The participation of these star members can help stimulate constructive discussions and informational exchanges between the users. Therefore, a greater number of these star members can result in a higher quality of the available pool of knowledge in the community. Thus,

H5: Users benefit more from user communities that have a larger number of star members.

\section{Exclusivity}

In the theory of clubs, individuals group together to share the cost of a public good. One of the underlying premises of club theory is that group size must be controlled for to ensure optimal sharing (Buchanan 1965). Hence, exclusion mechanisms are frequently used to monitor users' rates of utilisation and to bar nonmembers and/or nonpayers. It is considered that such exclusion mechanisms provide incentives for members to join, to contribute to the group and to pay dues (Cornes and Sandler 1996). It is therefore, proposed that

H6: Users benefit more from user communities that have exclusion mechanisms.

As exclusivity can take more than one form, the following subhypotheses are expected:

H6a: Users benefit less from user communities with memberships that are open to the general public.

H6b: Users benefit more from user communities that require their members to pay a membership fee.

\footnotetext{
${ }^{6}$ Three measures of success were analysed - products in development, products on the market and employment growth.

${ }^{7}$ GenBank is the NIH genetic sequence database, an annotated collection of all publicly available DNA sequences. (http://www.psc.edu/general/software/packages/genbank/genbank.html, accessed on 14th February 2006.)
} 


\section{Research Methodology}

Computer Associates' user groups were selected as the specific research context, to quantitatively analyse the above-mentioned hypotheses on the determinants of successful user communities. To assist in the formulation of the questionnaire, explorative interviews with twenty-nine user group presidents and members, as well as a CA employee who was responsible for the user group program, were conducted between October and December 2004. From the interviews, it was decided that a web-based questionnaire would be the ideal data-collection method.

Between May and June 2005, a pre-test of the questionnaire was carried out with seven individuals in CA user groups that use various CA software. On the 19th July 2005, an email with a link to the web-based survey was sent to 398 user group presidents and other committee members. These individuals were requested to participate in the survey and to forward the link to their members. Their contact details were obtained from the official CA user group website ${ }^{8}$ Reminder emails were then sent on the 2nd September 2005, the 11th October 2005 and the 21st November 2005. As of the 19th December 2005, a total of 1055 responses have been received from individuals in 203 different CA user groups.

To supplement the collected data, an additional interview with a CA employee was conducted on the 16th November 2005 with the specific aim to gain more information regarding the history of the CA user group program.

\section{Computer Associates' User Groups}

Computer Associates (CA) was founded in 1976 when Charles Wang decided to market a sorting program, CA-SORT for IBM mainframes (Campbell-Kelly 1995). Since then, CA has acquired more than seventy firms, ranging from medium-sized companies valued at a few million U.S. dollars up to larger companies such as Legent, which CA acquired at a cost of US\$ 1.8 billion in 1995 (Campbell-Kelly 2000). As a result of the numerous acquisitions, CA possesses today a diverse product portfolio and delivers products and services for operations, security, storage, life cycle and service management $9^{9}$, CA is currently one of the world's largest independent software vendors 10

\footnotetext{
${ }^{8} \mathrm{http}: / /$ causergroups.com/UserGroups/ (accessed on the 7th July 2005).

${ }^{9}$ See the Appendix for a brief overview of the various software products CA offers.

${ }^{10}$ Based on 2003 revenues, the OECD ranked CA fifth in the independent software vendor industry behind Microsoft Corp., Oracle Corp., SAP AG and Softbank Corp. (Houghton 2004). In the worldwide distributed performance and availability management software industry, CA had the fourth largest revenue in 2004 (Grieser 2005).
} 


\subsection{The CA User Group Program}

Through their many acquisitions, CA had also inherited a large number of user groups. During a discussion with some customers at a user conference in 1990, Charles Wang recognised the need to have a formal CA user group program to support his customers. The decision to establish this program was to provide CA users with a platform to communicate not only with other users but also with CA. This close communication was expected to bring learning benefits to the users. CA on the other hand was expected to gain not only from the user-input for the development process of their products but also from customer references who were willing to help them sell their products. Although CA wanted their user groups to be operated independently from them, they wanted to have the possibility to attend these user group meetings to occasionally conduct presentations on updates of their products and to listen in on the types of issues and concerns that the users discussed amongst themselves. Through the years, the user group program became more centralised with CA taking over a large proportion of the organisational planning for the user groups.

In the early nineties, the user group program consisted of around 20 security software groups and roughly 10 systems software groups. To date, there are over 300 user groups worldwide, each supporting any one or more of CA's software product categories.

To be an officially supported CA user group, user groups have to satisfy a number of requirements. Firstly, each group has to have a minimum of 20 members from at least eight companies 11 . Secondly, it is required that members meet physically at least once a year for a full-day meeting. Thirdly, although user groups have the freedom to choose their members, CA specifies that user groups are not allowed to be used for the solicitation for a member's personal or company benefit. Lastly, CA does not support the establishment of a new user group within a one hour commute of an existing CA user group that supports the same CA product/s.

Official CA user groups are entitled to logistical support from CA, such as the assistance in the organisation of user group activities; free refreshments and the use of CA facilities for user group meetings; as well as web administration support for their user group web sites. Furthermore, user group presidents are given free accommodation to attend the Computer Associates Regional Exchange (CARE) conference, which takes place 2 days before CA's official annual user conference, CA World. The CARE conference consists of a 2 day program where user group presidents not only have the opportunity to network with other IT professionals (i.e. other group leaders) and personally communicate with the product and development managers of their respective software products, but also have the chance to voice out their feedback and opinions to executives on the

\footnotetext{
${ }^{11}$ User groups established before April 2000 required a minimum of eight members from at least four companies.
} 
management board of CA. In addition, group leaders also receive a registration fee waiver to attend CA World.

\subsection{How CA Leverages its User Groups}

To provide an illustration of how CA leverages its user groups, this section will focus on one particular CA product, the life cycle management mainframe software - Endevor. The Inter-National User Group for Endevor (I-NUGE) ${ }^{12}$ is a group of Endevor users made up of representatives from individual local user groups from around the world. Since its establishment in 1992 2 , the I-NUGE has provided its members with a platform for the open exchange of knowledge, experiences, resources, and questions to organisations and individuals involved in the design and development of software applications using Endevor tools and related software. Furthermore, the I-NUGE also acts as an intermediary between CA and Endevor users by organising and prioritising enhancement and upgrade requests from its members and by providing a means of presenting a united position on various issues (when requested by the membership) to CA.

Each I-NUGE member not only represents an organisation that is in possession of a CA license of the Endevor software but also one of the 26 local Endevor user groups recognised by CA. Each local group may appoint up to three I-NUGE representatives, although only one vote is allowed per local user group regardless of the number of representatives from that group. Membership of the I-NUGE also includes a CA representative from the CA Endevor product group, whose involvement in the group is only upon request and as a non-voting participant. The executive board of the I-NUGE consists of a president, a vice-president, an administrator, an internet webmaster, a European liaison, a Asia-Pacific liaison, and the past president from the previous year.

One important function of the I-NUGE for CA is its coordination of the Demand Analysis Requests (DAR) process. DARs are change and/or fix requests submitted by users to CA for updating their software product. At the individual local user group level, members suggest and vote for DARs that they would like for CA to implement in their future releases. These locally prioritised DARs are then accumulated by the I-NUGE and further prioritised, this time at the international level. These internationally prioritised DARs are then submitted by the I-NUGE to CA. As compared to requests submitted by individual organisations, these "power in number" requests are significantly more influential. It should however be noted that CA exercises its discretion in deciding which of the submitted DARs to implement in future releases of their software.

\footnotetext{
${ }^{12}$ Information of this user group was obtained from the official I-NUGE website, http://www.inuge.com/ and from correspondences with various committee members of the I-NUGE.

${ }^{13}$ In 1992, the NUGE (National User Group for Endevor) was established. To better reflect its growing international membership, the name of the user group was changed to the Inter-National User Group for Endevor in 1996.
} 
Besides assisting in the transfer of user-specific information from the users to CA, the I-NUGE also disseminates firm-specific information from CA to its members. Examples of such information include updates of the development process of the next Endevor release, announcements of general technology update webcasts, as well as registration requests for beta testers and development buddies 14

Furthermore, CA also profits from the user-to-user support in the user groups. Instead of contacting the official CA support lines, individual users can browse forum archives of their local Endevor user groups or approach other members for assistance when they encounter difficulties with their software. Additionally, users can also browse the I-NUGE shareware website to access a compilation of user-generated add-ons, utlities and applications.

\section{Data Description}

Before the determinants of successful CA user groups can be identified, it is necessary to firstly provide a brief description of the dependent, independent and control variables to be used in the multivariate analysis.

\section{Dependent Variable}

\section{Time Saved}

The benefits users received from their user communities are measured by the perceived number of hours of personal work time they were able to save over the course of the last year. If users were uncertain of the approximate number of hours they saved, they had the possibility to indicate a range of hours.

If users indicated the number of hours they saved as an interval, time saved lower refers to the lower bound and time saved upper the upper bound of their indicated interval.

The variable time saved mean refers to the geometric mean ${ }^{15}$ of time saved upper and time saved lower. To take into account users who had indicated intervals where the lower bound was 0 , the following formula is used to calculate time saved mean,

$$
\text { Time saved mean }=\sqrt{(\text { time saved lower }+1)(\text { time saved upper })} \text {. }
$$

\footnotetext{
${ }^{14}$ The CA Development Buddy Program gives users the opportunity to work closely with CA developers on a specific functionality of the software.

${ }^{15}$ The geometric mean is smaller than or equal to the arithmetic mean, as it gives more weight to the lower as compared to the upper bound. This measure is more appropriate than the arithmetic mean, because the amount of time users saved is positively skewed. See Figure 1 for an illustration of this skewed distribution.
} 
To ensure consistency, it is also necessary to perform a similar transformation for users who responded with a point estimate, time saved point with the following formula,

$$
\text { Time saved mean }=\sqrt{(\text { time saved point }+1)(\text { time saved point })} \text {. }
$$

\section{Independent Variables}

Strong Ties

The number of strong ties is the number of close contacts (excluding colleagues) users have in their CA user group. Close contacts are defined as individuals with whom they communicate frequently, whom they can approach at any time for help and whom they would willingly help at any time.

\section{Time Spent}

The number of days individuals spent participating in formal user group activities in the course of the last year will be used as a proxy for an individual's investment in his learning ability. Four dummy variables are generated. The first dummy no participation takes the value 1 if individuals did not participate in any formal user group activities in the last year, and 0 otherwise. The next dummy time spent 1 takes the value 1 if individuals spent between 0 and 2 days, and 0 otherwise. The third dummy time spent 2 takes the value 1 if individuals spent between 3 to 6 days, and 0 otherwise. Finally, the last dummy time spent 3 takes the value 1 if individuals spent 7 days or more, and 0 otherwise.

\section{Face-to-Face Communication}

In the course of the last year, users could formally communicate with other members in the group through physical meetings; web, video and telephone meetings; as well as through the user group's forum. Two dummy variables are constructed. The first dummy face-to-face takes the value 1 if users interacted face-to-face with their user group in the last year and 0 otherwise. The second dummy no face-to-face takes the value 1 if users only interacted with their user groups through web, video and telephone meetings, and/or through the user group's forum; and 0 otherwise ${ }^{16}$

\section{Stars}

Users were asked to indicate their estimate of the number of highly qualified individuals in their CA user group, of whom they have learnt a lot from. Highly qualified individuals are defined as experienced and skilled users whom have a high knowledge of the CA software. To proxy for the number of stars in each user group, the average number of respondents' estimates per user group is used.

\section{Open Culture}

\footnotetext{
${ }^{16}$ These two dummy variables need not be perfectly multicollinear with each other, as there may exist users who did not participate in any formal user group activities. See the description of time spent for more details.
} 
Users were asked to indicate the extent to which they agreed or disagreed with the following statement: "This CA user group has been extremely successful in promoting an open exchange of information between its members." An open exchange of information is defined as the willingness of members to share and exchange information with one another. The open culture variable takes the value 1 when at least $50 \%$ of the members in each user group who responded to the survey indicated that they either strongly agreed or somewhat agreed with the statement, and 0 otherwise.

\section{Exclusivity}

Users who are on the management committee of their user group were asked to indicate how individuals can become members in their user groups. Two dummy variables are generated - one to control for the openness of a group's membership and the other for the existence of a membership fee. The first dummy open membership takes the value 1 if membership is open to the general public, and 0 otherwise ${ }^{17}$. The other dummy membership fee takes the value 1 if members are required to pay a membership fee, and 0 otherwise. If multiple differing answers were provided for each group, the answer of the group leader (i.e. president, co-president and/or vice-president) is used.

\section{Control Variables}

\section{Female}

To control for gender effects, this variable takes the values 1 for females and 0 otherwise.

\section{Learn}

Users who joined the user group with the intention to learn may have different expectations from the user community as compared to users who joined for other reasons. Users were asked to indicate the extent to which they agreed or disagreed with the following statement on why they joined their user group: "For the opportunity to learn from experienced users". Learn takes the value 1 if users indicated that they either strongly agreed or somewhat agreed with the statement, and 0 otherwise.

\section{Used code}

To control for individuals who made use of source code from other members in their user community this variable takes the value 1 if respondents indicated that they used source code of other members in their user groups, and 0 otherwise.

\section{Reciprocity}

Reciprocity is a social norm that may affect the benefits users are able to receive from their user communities. To control for this effect, reciprocity takes the value 1 if respondents indicated

\footnotetext{
${ }^{17} \mathrm{~A}$ more restrictive form of membership is to allow only individuals of firms who have a license of the CA product to be members.
} 
that they either strongly agreed or somewhat agreed with the following statement: "I help other members because I received help from this CA user group in the past", and 0 otherwise.

\section{Committee}

User group presidents and other committee members invest additional time managing and organising activities for their user groups. To control for this extra time, which is not accounted for in the time spent variable, the committee variable takes the value 1 if the user is a committee member of his user group and 0 otherwise.

\section{Contribute}

Individuals can contribute to the user group by sharing information on their use experience with the CA software. They can do this by making a presentation, by participating in discussion-sessions or by making posts on the user group's forum. Users were asked to indicate the extent to which they agreed or disagreed with the following statement: "I reveal information on how my company utilises the CA software because I benefit from the resulting discussions." This variable takes the value 1 if respondents indicated that they either strongly agreed or somewhat agreed with the statement, and 0 otherwise.

\section{Use Experience}

To control for the level of experience of users, users were asked to indicate the year in which they started using their most important CA software that they frequently used at their present employment. The use experience variable is constructed by subtracting their indicated year from 2006.

\section{Empirical Analysis}

\subsection{Descriptive Statistics}

Table 1 presents the descriptive statistics of the above mentioned dependent, independent and control variables. The final sample consists of 924 responses from individuals in 161 different user groups ${ }^{18}$. The number of responses per user group ranges from 1 to 40 , with at least $87.12 \%$ of the respondents belonging to user groups from which at least 4 responses were received. It can be seen that users are able to save an average of 22.29 number of hours per year ${ }^{19}$. The minimum number of hours they saved is 0 and the maximum ${ }^{20} 600.26 .62 \%$ of the respondents did not save any time

\footnotetext{
${ }^{18}$ Although a total of 1055 responses were received, only 924 were completely filled out with respect to the abovementioned variables.

${ }^{19}$ Approximately $22 \%$ of the respondents indicated the amount of hours they saved as an interval.

${ }^{20}$ The maximum reported here is the time saved point estimate of 600 hours.
} 
Figure 1: Number of hours saved per year $(\mathrm{N}=924)$

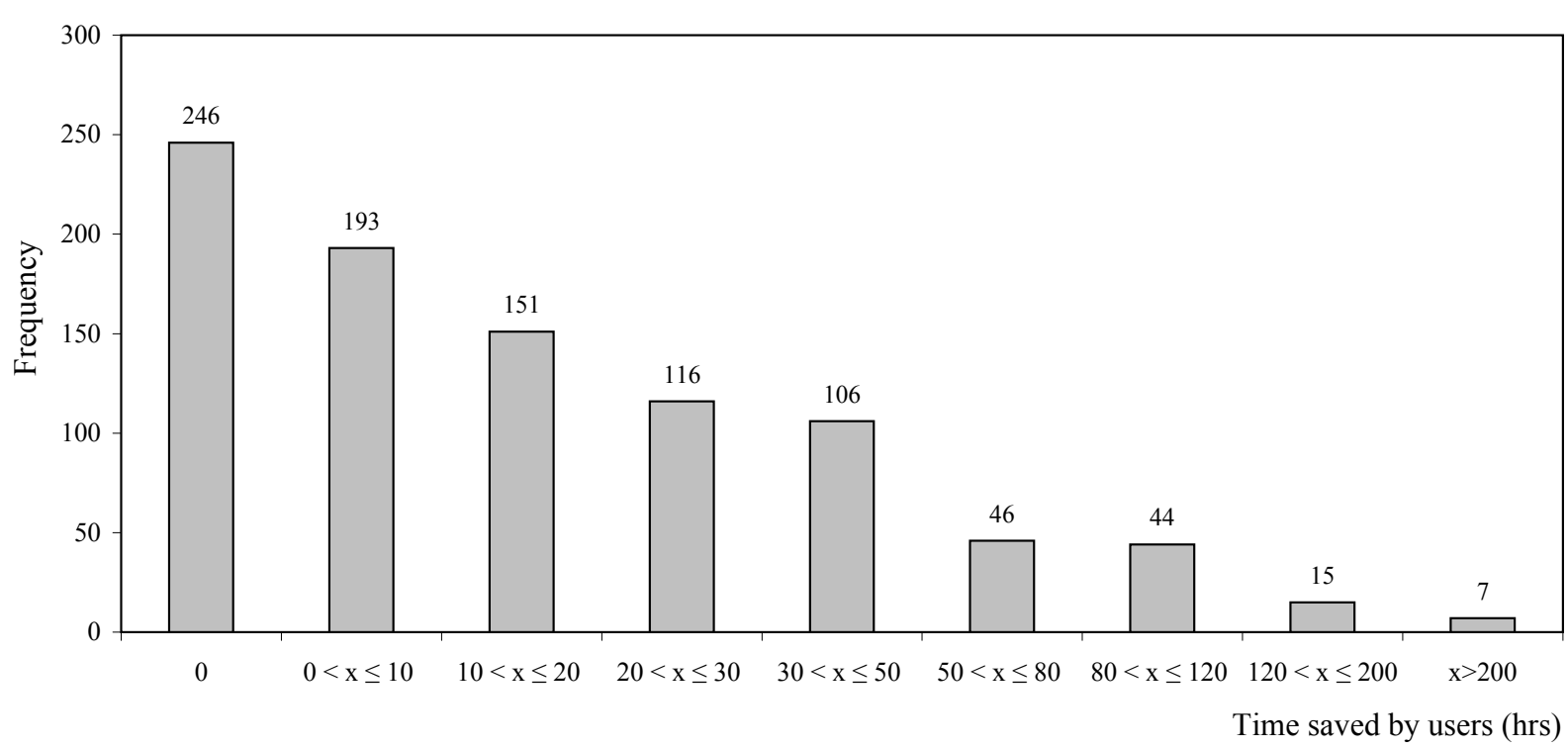

in the last year. An observation of the first to fourth percentiles of time saved mean suggests that the variable has a positively skewed distribution. Figure 1 illustrates this skewed distribution 21 .

CA user groups also appear to provide a platform for users to network and to make valuable social contacts within their user communities. Specifically, users have on average 3.49 number of strong ties in their user groups. This number ranges between 0 to 200 . Approximately $40 \%$ of the respondents do not have any close contacts in their user groups. Similar to the time saved mean variable, the first to fourth percentiles of the number of strong ties also indicate a positively skewed distribution. Of the 924 respondents, $22 \%$ did not participate in any formal user group activities in the last year. Majority of the users spent less than a week with their user communities, with 39\% having spent between 0 and 2 days, 30\% between 3 and 6 days, and only 10\% having invested 7 days or more in their user groups. Face-to-face interaction seems to be the most popular means of communication between users and their user groups as $73 \%$ of respondents ${ }^{22}$ had attended physical user group meetings. This finding is not surprising as CA requires its user groups to meet at least once a year for a full-day meeting. $5 \%$ of the respondents participated only in the user group's forum and/or in web, video and telephone meetings in the last year.

The next four variables are user group-specific characteristics. $87 \%$ of the respondents belong to CA user groups that have an open culture of communication. As displayed in Table 2, 84\% of the 161 user groups have members who are willing to share and exchange information with one

\footnotetext{
${ }^{21}$ The number of hours presented here refer to the time saved mean variable as described in Section 6

${ }^{22} 93 \%$ of all participants who participated in formal user group activities $(\mathrm{N}=719)$ interacted with their user groups face-to-face.
} 
Table 1: Descriptive Statistics I - Across Individuals $(\mathrm{N}=924)$

\begin{tabular}{|l|r|r|c|c|}
\hline \hline Variable & Mean & S.D. & Min. & Max. \\
\hline time saved mean & 22.29 & 38.53 & 0 & 600.5 \\
\hline time saved lower* & 20.49 & 37.65 & 0 & 600 \\
\hline time saved upper* & 25.31 & 51.60 & 0 & 1000 \\
\hline strong ties & 3.49 & 8.81 & 0 & 200 \\
\hline time spent & & & & \\
time spent 1 (0/1) & 0.39 & & 0 & 1 \\
time spent 2(0/1) & 0.30 & & 0 & 1 \\
time spent 3 (0/1) & 0.10 & & 0 & 1 \\
\hline no participation (0/1) & 0.22 & & 0 & 1 \\
\hline face-to-face (0/1) & 0.73 & & 0 & 1 \\
\hline no face-to-face (0/1) & 0.05 & & 0 & 1 \\
\hline open culture (0/1) & 0.87 & & 0 & 1 \\
\hline stars & 6.78 & 4.07 & 0 & 25 \\
\hline open membership (0/1) & 0.37 & & 0 & 1 \\
\hline membership fee (0/1) & 0.17 & & 0 & 1 \\
\hline female (0/1) & 0.22 & & 0 & 1 \\
\hline learn (0/1) & 0.93 & & 0 & 1 \\
\hline used code (0/1) & 0.21 & & 0 & 1 \\
\hline reciprocity (0/1) & 0.57 & & 0 & 1 \\
\hline committee (0/1) & 0.28 & & 0 & 1 \\
\hline contribute (0/1) & 0.74 & & 0 & 1 \\
\hline use experience & 10.57 & 6.40 & 1 & 34 \\
\hline
\end{tabular}

${ }^{*}$ For point estimates, time saved lower has the same value as time saved upper.

another. Table 2 also shows that the average number of star members in each user group is 6.38 with values ranging from 0 to 25 . Similarly, the mean of the number of stars of users' user groups (from Table 1) is $6.78 .37 \%$ of the respondents are in user groups that have an open membership structure that allows anyone interested (i.e. from the general public) to become a member. From Table 2, it can be seen that $35 \%$ of the 161 user groups have such an open membership structure. Finally, only $13 \%$ of the user groups require their members to pay a membership fee; $17 \%$ of the respondents are in such user groups.

$22 \%$ of the respondents are female. Consistent with the definition of user communities as introduced in Section 3, 93\% of the respondents indicated that they joined their user groups with the aim 
Table 2: Descriptive Statistics II - Across User Groups ( $\mathrm{N}=161)$

\begin{tabular}{|l|r|c|c|c|}
\hline \hline Variable & Mean & S.D. & Min. & Max. \\
\hline open culture $(0 / 1)$ & 0.84 & & 0 & 1 \\
\hline stars & 6.38 & 4.38 & 0 & 25 \\
\hline open membership (0/1) & 0.35 & & 0 & 1 \\
\hline membership fee $(0 / 1)$ & 0.13 & & 0 & 1 \\
\hline
\end{tabular}

to learn. Users were also asked if they had utilised source code written by other users in their user group. $21 \%$ indicated that they make use of add-ons, utilities, applications and/or exits from their user community. Majority of the respondents seem to exhibit the social norm reciprocity, as $57 \%$ of the respondents indicated that they were willing to help other users because they had received assistance from their user group in the past. $28 \%$ are committee members in their user groups. This finding is not surprising as these individuals are more committed and interested to participate in a survey regarding their user group. Another reason for this relatively high percentage could also be because committee members were contacted directly to participate in the survey, whereas regular user group members were informed of the survey indirectly through their committee members. As $74 \%$ of the respondents contribute to their user communities, the free-rider problem (introduced in Section 2) does not seem to be a substantial problem in majority of the CA user groups surveyed. Finally, the average number of years that users have had experience with their CA software is 10.57 and there appears to be a relatively diverse level of use experience amongst the users as can be seen from the first percentile ( 6 years), the second percentile ( 9 years), the third percentile (15 years) and the $95 \%$ percentile (23 years). This result suggests that CA user groups provide a platform for newer users to learn from more experienced individuals. A closer inspection of this variable at the user group level reveals that majority of the user groups have users with a relatively heterogeneous level of use experience.

Table 3 presents an overview of the bivariate relationships between the independent and control variables ${ }^{23}$ It can be seen that with the exception of 4 correlation coefficients, all the rest of the coefficients are below 0.25 . These stronger interrelationships will be elaborated in the following. Firstly, there appears to be a negative relationship $(\rho=-0.35)$ between respondents who do not participate and those who invest between 3 and 6 days in user group activities per year. As these 2 variables have dichotomous values that represent nominal data, this negative relationship is a result of the approximately equal numbers of individuals who did not participate $(\mathrm{N}=236)$ and those who

\footnotetext{
${ }^{23}$ The variables time spent 1 and face-to-face are omitted in the table, as these 2 variables will not be included in the multivariate analysis in Section 7.2 .
} 
-19-

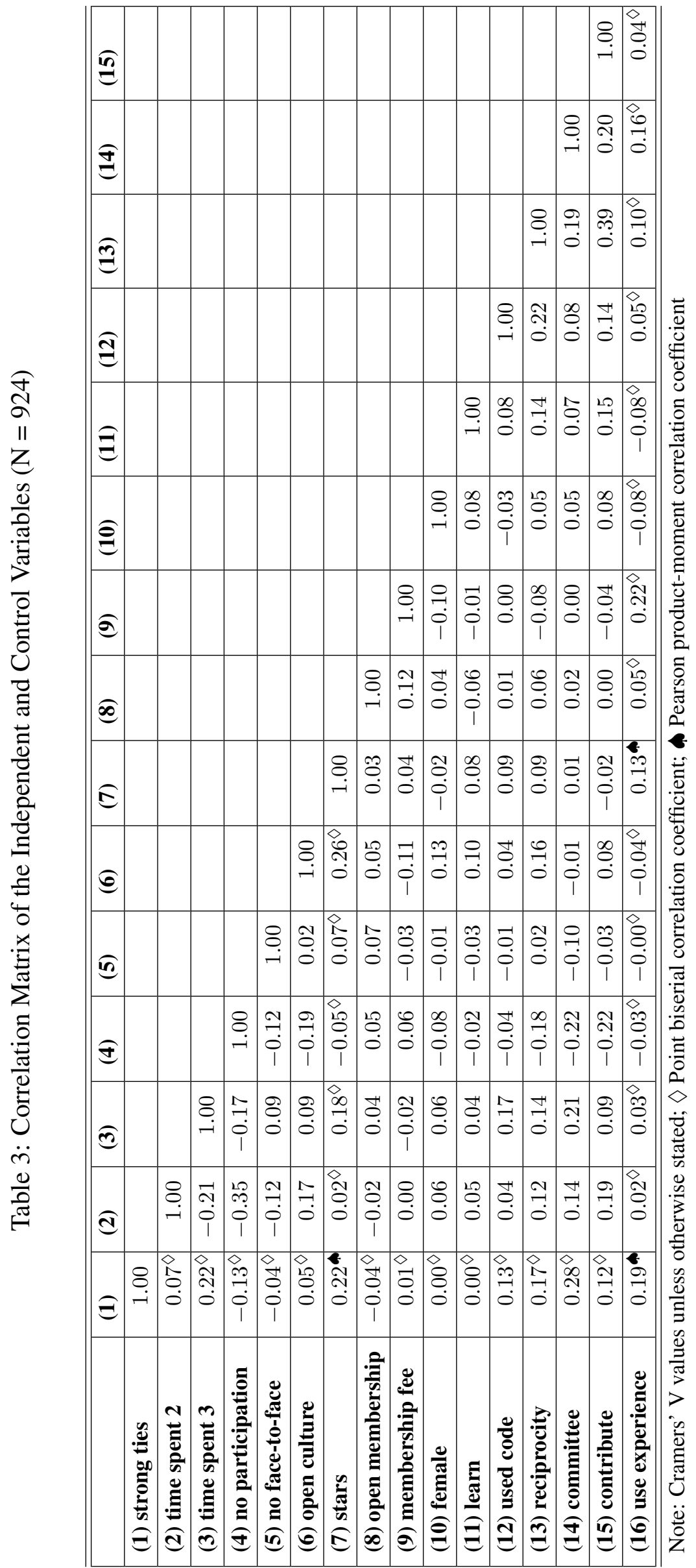


spent between 3 and 6 days $(\mathrm{N}=314)$ in formal user group activities. Next, committee members appear to have a larger number of strong ties to other members in their user groups $(\rho=0.28)$. This relationship is reasonably conceivable as committee members play a more central role in the user group ${ }^{24}$. They are more accessible to the full member contact list and have more interactions with individual members when organising user group activities.

Thirdly, it is not surprising that respondents who exhibit the social norm reciprocity are also those who contribute to the user group ( $\rho=0.39$ ), as individuals who have benefitted from the user group in the past would be more willing to contribute to their user group by giving oral presentations, assisting members in solving their difficulties, participating in discussion-sessions or in answering posts on the user group's forum. Finally, the number of star members and the openness of the communication culture of each users' user group are positively related $(\rho=0.26)$. To interpret this positive relationship, it is also necessary to see how these 2 variables are correlated at the user group level. The Cramer's V value at the user group level $(\rho=0.23)$ is fairly similar to that at the individual level. These findings suggest that the participation of more star members may help promote an open culture of communication; and/or that as star members may be interested in interacting with other users, they are motivated to join user groups whose members are more open to share and exchange information with one another.

\subsection{Multivariate Analysis}

To empirically test the hypotheses introduced in Section 3 , this paper will apply a pooled interval estimation method with robust standard errors in the presence of intra-cluster correlation. First, a short overview of the estimation method is presented. This is followed by the empirical analysis of the estimated models.

\subsubsection{Estimation Method}

As the analysis uses data from individuals in different user groups, there is a high probability that responses from individuals within the same user group may be correlated, possibly through unobserved cluster effects (Wooldridge 2002). This intra-cluster correlation is often referred to as over-dispersion or extra variation in an estimated statistic beyond what would be expected under independence. Hence, analyses that assume independence of the observations will tend to underestimate the true variance, which would lead to test statistics with inflated Type I errors (Williams 2000). To avoid this over-dispersion problem in ordinary least squares (OLS) regressions, unbiased variance estimators can be computed (Rogers 1993).

\footnotetext{
${ }^{24}$ See Wasserman and Faust 1994 for a more detailed description of centrality in social networks.
} 
Let the model be

$$
y=X \beta+\varepsilon,
$$

where $y$ represents continuous outcomes of time saved and $X$ the dependent and control variables. The model assumes $\epsilon \sim N\left(0, \sigma^{2} I\right)$. To estimate $\beta$, the OLS method minimises the sum of squared errors $\left(\varepsilon^{\prime} \varepsilon\right)$, where

$$
\varepsilon^{\prime} \varepsilon=(y-X \beta)^{\prime}(y-X \beta) .
$$

The OLS estimate of $\beta$ is

$$
b=\left(X^{\prime} X\right)^{-1} X^{\prime} y
$$

and its variance is

$$
\operatorname{var}(b)=E\left(X^{\prime} X\right)^{-1} X^{\prime}(y-E y)(y-E y)^{\prime} X\left(X^{\prime} X\right)^{-1} .
$$

Under the assumption that the observations are independently and identically distributed, this variance reduces to $\left(X^{\prime} X\right)^{-1} \sigma^{2}$. In the presence of intra-cluster correlation this assumption is relaxed, which allows for non-identical diagonal terms as well as nonzero off-diagonal terms. However, as observations from different clusters are assumed to be independent, these off-diagonal terms can only take on nonzero values if observations come from the same cluster. These nonzero terms are then represented by the appropriate products of the residuals (Rogers 1993).

Approximately $22 \%$ of the respondents indicated the number of hours they saved in the last year as an interval. One possibility to analyse such interval data is to estimate a pooled OLS regression on the arithmetic or geometric mean of these intervals. The drawback of this OLS method is that the analysis would not reflect any uncertainty concerning the nature of the exact values within each of these intervals 25 . Hence analysing the data with an interval regression - a slightly modified version of the standard censored tobit model (Tobin 1956) - would provide more accuracy to the estimation results. An advantage of the interval regression over the OLS estimation method is that it is able to estimate the $\beta$ coefficients even if the dependent variable $y$ is non-observable, provided the interval in which $y$ falls is observable (Wooldridge 2002).

Assuming the same model as in Equation (1), $y$ is now defined as continuous outcomes which are either observed or unobserved. For observations $j \in \zeta, y_{j}$ is observed as point outcomes. Observations $j \in \theta$ are intervals, such that $y_{j}$ is in the interval $\left[y_{1 j}, y_{2 j}\right]$. The interval regression is estimated using the maximum likelihood method. The log likelihood to be maximised is

\footnotetext{
${ }^{25}$ www.ats.ucla.edu/stat/stata/dae/intreg.htm (accessed on 4th April 2006)
} 


$$
\begin{aligned}
\ln L= & -\frac{1}{2} \sum_{j \in \zeta}\left\{\left(\frac{y_{j}-x \beta}{\sigma}\right)^{2}+\log 2 \pi \sigma^{2}\right\} \\
& +\sum_{j \in \theta} \log \left\{\Phi\left(\frac{y_{2 j}-x \beta}{\sigma}\right)-\Phi\left(\frac{y_{1 j}-x \beta}{\sigma}\right)\right\}
\end{aligned}
$$

where $\Phi()$ is the standard cumulative normal. ${ }^{26}$ Similar to the OLS regression method, unbiased variance estimates can also be computed for the interval regression to control for intra-cluster correlation.

\subsubsection{Empirical Results}

Table 4 presents the results of the above-mentioned estimation method. The logarithmical forms of the number of hours saved, the number of strong ties and the number of star members will be used in the analysis. To accommodate for 0 values, the logarithm of 1 plus the values of each variable is used.

To test for the presence of heteroscedasticity in Model 1, the $\sigma$ term was expressed in the loglikelihood function of Equation (5) as a set of all 16 independent and control variables. A likelihoodratio test is then conducted with this unrestricted model and the restricted model where $\sigma$ is kept constant (i.e. homoscedasticity). The likelihood-ratio test statistic is 32.21 with 16 degrees of freedom and a p-value of 0.0094 . This result indicates that the null hypothesis of homoscedasticity can be rejected at the $1 \%$ level of significance. Hence, heteroscedasticity-robust standard errors in the presence of intra-cluster correlation are estimated to control for both heteroscedasticity and cluster effects.

As can be seen from Model 1 in Table 4, the number of strong ties users have in their user groups is positively significant at the $1 \%$ level ${ }^{27}$ In particular, when a user's number of strong ties doubles, the number of hours he can save increases by approximately $22.4 \%$. Hence, the results support hypothesis 1 that users benefit more from a greater number of strong relational ties as this increases the number of channels from which they can receive relevant information and knowledge.

As a proxy for an individual's investment in his learning abilities, 3 dummy variables on the amount of time the user invests in participating in user group activities are included. The reference group are users who participate between 0 and 2 days per year. Results show that individuals who do

\footnotetext{
${ }^{26}$ STATA 9 Reference A-J, 2005. Note that the right- and left-censored outcomes have been omitted for simplicity.

${ }^{27}$ Models 1 and 2 are also estimated with the pooled OLS method, with time saved mean as the dependent variable. Coefficient estimates and their significance levels were very similar to those from the interval estimation method.
} 
Table 4: Interval Regression Models with Heteroscedasticity-Robust Standard Errors in the Presence of Intra-Cluster Correlation

\begin{tabular}{|c|c|c|}
\hline & Model 1 & Model 2 \\
\hline & $\begin{array}{l}\text { ln (time saved lower }+1) \\
\ln (\text { time saved upper }+1)\end{array}$ & $\begin{array}{l}\ln (\text { time saved lower }+1) \\
\ln (\text { time saved upper }+1)\end{array}$ \\
\hline \multirow[t]{2}{*}{$\ln ($ strongties +1$)$} & $0.224^{* * *}$ & $0.249^{* * *}$ \\
\hline & {$[0.060]$} & {$[0.065]$} \\
\hline \multirow[t]{2}{*}{ time spent 2 ( 3 to 6 days) } & $0.268^{* *}$ & $0.226^{*}$ \\
\hline & {$[0.121]$} & {$[0.132]$} \\
\hline \multirow[t]{2}{*}{ time spent 3 ( 7 or more days) } & $0.663^{* * *}$ & $0.506^{* *}$ \\
\hline & {$[0.210]$} & {$[0.229]$} \\
\hline \multirow[t]{2}{*}{ no participation } & $-0.375^{* * *}$ & $-0.361^{* * *}$ \\
\hline & {$[0.119]$} & {$[0.125]$} \\
\hline \multirow[t]{2}{*}{ no face-to-face } & -0.065 & -0.085 \\
\hline & {$[0.164]$} & {$[0.170]$} \\
\hline \multirow[t]{2}{*}{ open culture } & $0.274^{* *}$ & $0.207^{*}$ \\
\hline & {$[0.111]$} & {$[0.120]$} \\
\hline \multirow[t]{2}{*}{$\ln (\operatorname{stars}+1)$} & $0.206^{* *}$ & $0.256^{* *}$ \\
\hline & {$[0.102]$} & {$[0.105]$} \\
\hline \multirow[t]{2}{*}{ open membership } & $0.146^{*}$ & $0.174^{* *}$ \\
\hline & {$[0.076]$} & {$[0.079]$} \\
\hline \multirow[t]{2}{*}{ membership fee } & $-0.207^{* *}$ & $-0.169^{* *}$ \\
\hline & {$[0.084]$} & {$[0.079]$} \\
\hline \multirow[t]{2}{*}{ female } & $-0.415^{* * *}$ & $-0.445^{* * *}$ \\
\hline & {$[0.098]$} & {$[0.103]$} \\
\hline \multirow[t]{2}{*}{ learn } & $0.772^{* * *}$ & $0.677^{* * *}$ \\
\hline & {$[0.184]$} & {$[0.197]$} \\
\hline \multirow[t]{2}{*}{ used code } & $0.817^{* * *}$ & $0.846^{* * *}$ \\
\hline & {$[0.095]$} & {$[0.106]$} \\
\hline \multirow[t]{2}{*}{ reciprocity } & $0.524^{* * *}$ & $0.511^{* * *}$ \\
\hline & {$[0.100]$} & {$[0.107]$} \\
\hline \multirow[t]{2}{*}{ committee } & -0.136 & $-0.188^{*}$ \\
\hline & {$[0.095]$} & {$[0.104]$} \\
\hline \multirow[t]{2}{*}{ contribute } & $0.260^{* *}$ & $0.294^{* *}$ \\
\hline & {$[0.122]$} & {$[0.128]$} \\
\hline \multirow[t]{2}{*}{$\ln$ (use experience) } & 0.001 & 0.022 \\
\hline & {$[0.061]$} & {$[0.065]$} \\
\hline \multirow[t]{2}{*}{ constant } & -0.06 & -0.104 \\
\hline & {$[0.245]$} & {$[0.262]$} \\
\hline Observations & 924 & 805 \\
\hline Min. obs per group & 1 & 4 \\
\hline No. of user groups & 161 & 92 \\
\hline Log pseudolikelihood & -1652.259 & -1433.163 \\
\hline
\end{tabular}

Robust standard errors in brackets

$*$ significant at $10 \% ; * *$ significant at $5 \% ; * * *$ significant at $1 \%$ 
not participate in user group activities receive 37.5\% less benefits from their user groups as compared to individuals who invest between 0 and 2 days per year; this result is significant at the $1 \%$ level. Individuals who spend 3 days or more engaging in user group activities are able to save significantly more time; the individual effects of time spent 2 and time spent 3 are significant at the $5 \%$ and $1 \%$ level respectively. To test if an individual's benefits increases when he invests more time to improve his learning abilities (hypothesis 2), it is necessary to conduct a joint-test with the following null hypotheses:

$$
H_{0}:\left[\begin{array}{l}
\text { no part }=0 \\
\text { time spent } 2=0 \\
\text { time spent } 2=\text { time spent } 3
\end{array}\right]
$$

The chi-squared statistic of this joint-test is 26.16 with 3 degrees of freedom, and a p-value of 0 . This means that the joint null hypotheses can be rejected at the $1 \%$ level of significance. It can hence be concluded that users who spend more time with their user groups, i.e. invest more in their learning abilities, benefit more from their user communities. As a result, hypothesis 2 can be verified by the data.

Although the coefficient of the no face-to-face variable has a negative sign, the effect is not significant. This indicates that individuals who only interact with their user groups either through their user group's forum and/or through web, video and telephone meetings do not benefit less from their user groups as compared to individuals who participate in physical meeting ${ }^{28}$. It should however be noted that as only $6.54 \%$ of users who did engage in user group activities (i.e. only 47 respondents) did not have face-to-face interaction with other members in their user communities, the power of the above-mentioned joint-test is relatively small. Nevertheless, hypothesis 3 cannot be confirmed with the data.

Users in user groups with an open culture of communication are able to save $27.4 \%$ more hours as compared to users in user groups whose members are hesitant to share and exchange information with one another. When the number of star members in a user group doubles, users are able to increase the number of hours they save by approximately $20.6 \%$. Both these group-specific determinants are significant at the 5\% level. Hence, the data provides evidence that hypothesis 4 and 5 can be validated.

Individuals who are in user groups with an open membership save $14.6 \%$ (significant at the $10 \%$ level) more hours per year as compared to individuals who are in user groups whose memberships

\footnotetext{
${ }^{28} 78.7 \%$ of respondents who participated in physical meetings also participated in their user group's forum and/or in web, video and telephone meetings.
} 
are not open to the public. Hence, hypothesis 6 a cannot be confirmed by the data. Next, users who have to pay a membership fee save $20.7 \%$ (significant at the $5 \%$ level) less hours as compared to users in user groups that do not charge membership fees. Thus, hypothesis $6 \mathrm{~b}$ is also not supported by the data. A joint-hypothesis test of the significance of both these variables further concludes that they are jointly significant. The chi-squared statistic of this joint-test is 9.49 with 2 degrees of freedom, and a p-value of 0.0087 . These results provide evidence that at the $1 \%$ level of significance, individuals in user groups with less restrictive membership requirements are able to benefit more from their user groups. Hypothesis 6 is thus invalidated by the data.

In addition to the dependent variables, 7 additional control variables are also included in the estimation. Results show that females save significantly less hours as compared to males. Individuals who had joined their user groups with the intention to learn are able to save more than individuals who had joined their communities for other reasons. In comparison to users who do not utilise source code from other members in their community, users who do are able to save significantly more hours. Users who exhibit reciprocal behaviour are also able to save more hours than those who do not have this reciprocity characteristic. Additionally, users who contribute to their user groups are also able to benefit more from them. Finally, committee members and more experienced users do not significantly benefit more (or less) from their user communities. A joint-hypothesis test is conducted to test for the joint-significance of all the control variables. The chi-squared statistic of this test is 255.27 with 7 degrees of freedom, and a p-value of 0 . Hence, all 7 control variables are jointly significant.

To check the reliability and robustness of the results from Model 1, the sample is reduced to include only user groups from which at least 4 responses were received. This reduction in the sample also seeks to avoid potential endogeneity problems that may arise, as group specific variables such as the number of stars in each group and the openness of the group will now be based on at least 4 members of each group. Model 2 provides the results of the pooled interval regressions with heteroscedasticity-robust standard errors in the presence of intra-cluster correlation of 805 responses from individuals in 92 different user groups. It can be seen that the results are relatively similar to those of Model 1. Hypothesis 1 is still significantly supported by the data at the $1 \%$ level. A joint-hypothesis tes ${ }^{29}$ validates hypothesis 2 at the $1 \%$ level. Hypothesis 3 still cannot be confirmed. Hypothesis 4 can be verified at the $10 \%$ level and hypothesis 5 at the $5 \%$ level. Lastly, hypothesis $6 a, 6 b$ and 6 are again invalidated ${ }^{30}$ by the data.

\footnotetext{
${ }^{29}$ The chi-squared statistic of this joint-test is 19.51 with 3 degrees of freedom, and a p-value of 0.0002 .

${ }^{30} \mathrm{The}$ chi-squared statistic of this joint-test is 9.50 with 2 degrees of freedom, and a p-value of 0.0086 .
} 


\subsection{Management Implications}

This paper will now present four key findings of the empirical results for the successful management of user communities.

\section{Social Bonding Activities}

An essential characteristic of a successful user community is the existence of a social network. It has been shown that users who have a greater number of close contacts (i.e. strong ties) within their communities and who exhibit reciprocal behaviour benefit more from their user groups. Although it was not possible to validate the hypothesis that face-to-face interaction is more beneficial than other forms of virtual communication (due to the low power of the test), face-to-face meetings are a very good means of stimulating social interactions between members. For instance, members can get to know each other better through social events or functions that may take place before, between or after formal group meetings. Alternatively, more user-to-user contact can be stimulated through longer or more frequent intermission breaks between presentations at physical user group meetings. Such social bonding activities may not only promote social norms such as reciprocity and trust but can also facilitate the establishment of close contacts between group members.

\section{Quality and Frequency of Informational Exchanges}

User group meeting programs should consist of topics and issues that majority of the members are interested in and that are of a high quality as this would motivate more members to participate. Moreover, an active user group forum that has a regular exchange of knowledge between members will also motivate users to invest time to read and make posts on their group's forum (Lazar and Preece 2002). This is important because the empirical results indicate that users who spend more time engaging in group activities are able to benefit more from their groups.

\section{Incentive Scheme for Stars}

Results provide evidence that users in user groups with a larger number of star members are able to benefit more from their communities. This suggests that user communities can be used as a platform for the transfer of knowledge not only from more experienced stars to less experienced users, but also between experienced stars. Hence, one could provide incentives to attract and motivate highly experienced individuals (stars) to join and actively participate in user communities. There are various ways in which such a scheme can be implemented, either user- or firm-initiated. For instance, star members (as voted by the group) may receive a complementary voucher for a meal or a drink, sponsored by the rest of the members. Firms can also introduce incentives for star members, such as to provide more customised support or a reduced fee of the product to companies of star members. Alternatively, firms can recognise a star's participation in the community by inviting the star to be part of an executive user committee that is involved in the decision making 
process of future development strategies for the product ${ }^{31}$

\section{Open Membership and Communication}

Results propose that user communities should be open to the general public and have less restrictive membership structures. Individuals in user groups that do not impose a membership fee and/or allow interested individuals from the public to become members, benefited more from their communities. A less restrictive membership structure may increase the potential knowledge pool available by allowing a wider diversity of individuals to participate and contribute to the community. Additionally, the empirical results also suggest that there should be a free flow of knowledge and information within user communities. Hence, users should be encouraged to share their ideas, insights and experiences with each other.

\section{Conclusion}

By comparing the amount of time individuals in different user groups were able to save over the course of the last year, the aim of this study is to identify the determinants of successful user communities. Empirical findings demonstrate that the following determinants are relevant individualspecific factors: the number of strong intra-group contacts a user has and the amount of time users invest in their user groups. Furthermore, results also propose that user communities with an open communication culture, a larger number of star members and less restrictive membership requirements are more successful.

As user communities are voluntary in nature, rational individuals will not participate in and contribute to these groups unless they perceive a net benefit. Hence, the successful organisation of these communities is essential for members to be able to constantly leverage their user network for their own benefit. This paper proposes the following four implications for the management of user communities. Firstly, group leaders are advised to give users more opportunity to socially interact with each other at a more personal level. Secondly, it is essential that members not only have regular contact with one another throughout the course of the year, but that group leaders and forum moderators ensure that the knowledge and information exchanged within the group be of a high quality and relevance for majority of the users. Thirdly, it may be advisable to introduce an incentive for highly experienced and knowledgable users to join and participate in user group meetings, as their presence may increase the potential competence and attractiveness of the group. Lastly, user communities should not only be more open to the general public and have less restrictive membership structures, but also possess an open culture of communication.

\footnotetext{
${ }^{31}$ An example of such a committee is CA's Product Advisory Councils, which consist of product-specific groups of experienced, prominent IT and business professionals that help guide CA in its future offerings.
} 
Although these implications have been based on user groups associated to a proprietary software company, there exists sufficient group heterogeneity (due to the diversity of products CA provides and the large number of acquisitions CA made in the past) to be able to generalise these results for similar forms of user communities where users voluntarily group together to learn from each other. However, more industry-specific information will be needed before these implications can be applied to industries outside the software industry.

Nevertheless, the above-mentioned implications for the management of user communities provides a first insight into how groups of users can be organised. This knowledge is of particular importance for firms that intend to leverage user communities for innovation management. As illustrated in Section 5.2, a well managed user community can be used to efficiently organise and prioritise the pool of user information regarding their needs, feedback to existing products as well as ideas for new products. Furthermore, an open exchange of information and knowledge within these user communities can help enhance the creativity and innovative potential of individual users, which may result in user innovations (Shah 2005). Under certain conditions, firms may even be able to harness these user innovations and integrate them into their own proprietary solutions (Mayrhofer 2005).

A symbiotic relationship between firms and their user communities can provide conducive conditions for firms to constantly leverage their communities for innovation management (Dahlander and Magnusson 2005). This study has focused its analysis on the benefits users can obtain from their user communities. Future research should be directed towards investigating the integration of user communities into firms' research and development teams and examining the benefits firms are able to obtain from this integration. More research is still necessary before a framework for the optimal integration of user communities into firms' product development processes can be developed. One possibility could be to use user communities to identify leading-edge users, who have been shown to be essential sources of innovation (Urban and von Hippel 1988). In particular, user communities may be able to help reduce the search costs involved in finding these leading-edge users as well as in reducing the uncertainty of their dedication and commitment to cooperate with the firm. 


\section{References}

Bitzer, J., Schrettl, W., and Schröder, P. J. H. (2004). Intrinsic Motivation in Open Source Software Development. Nr. 2004/19, Diskussionsbeiträge des Fachbereichs Wissenschaft der Freien Universität Berlin.

Buchanan, J. M. (1965). An Economic Theory of Clubs. Economica, 32:1-14.

Campbell-Kelly, M. (1995). Development and Structure of the International Software Industry, 1950-1990. Business and Economic History, 24(2):73-110.

Campbell-Kelly, M. (2000). From Airline Reservations to Sonic the Hedgehog. A History of the Software Industry. MIT Press, Cambridge-London.

Carpenter, D., Esterling, K., and Lazer, D. (2003). The Strength of Strong Ties. A Model of Contact-Making in Policy Networks with evidence from U.S. Health Politics. Rationality and Society, 15(4):411-440.

Chakravorti, B. (2004). The New Rules for Bringing Innovations to Market. Harvard Business Review, 82(3):59-67.

Chesbrough, H. W. (2003). The Era of Open Innovation. MIT Sloan Management Review, 44(3):35-41.

Cohen, W. M. and Levinthal, D. A. (1990). Absorptive capacity: A new perspective on learning and innovation. Administrative Science Quarterly, 35:128-152.

Cohendet, P. (2006). Knowing Communities in Organizations. In Kahin, B. and Foray, D., editors, Advancing Knowledge and The Knowledge Economy, pages 91-109. MIT Press, CambridgeLondon.

Cornes, R. and Sandler, T. (1996). The Theory of Externalities, Public Goods and Club Goods. Cambridge University Press, Cambridge UK, second edition.

Cowan, R., Davis, P. A., and Foray, D. (2000). The Explicit Economics of Knowledge Codification and Tacitness. Industrial and Corporate Change, 9(2):211-253.

Crowston, K., Wei, K., Li, Q., and Howison, J. (2006). Core and Periphery in Free/Libre and Open Source Software Team Communications. In HICSS, Los Alamitos, CA, USA. IEEE Computer Society.

Dahlander, L. and Magnusson, M. G. (2005). Relationships between open source software companies and communities: Observations from Nordic firms. Research Policy, 34(4):481-493. 
Dougherty, K. L. (2003). Public goods theory from eighteenth century political philosophy to twentieth century economics. Public Choice, 117:239-253.

Ellis, H. C. (1965). The Transfer of Learning. MacMillan, New York.

Estes, W. K. (1970). Learning Theory and Mental Development. Academic Press, New York.

Fielding, R. T. (1999). Shared Leadership in the Apache Project. Communications of the ACM, 42(4):42-43.

Foray, D. (2004). The Economics of Knowledge. The MIT Press, Cambridge-London.

Franke, N. and Shah, S. (2003). How Communities Support Innovative Activities: An Exploration of Assistance and Sharing Among End-Users. Research Policy, 32(1):157-178.

Friedman, D. and McAdam, D. (1992). Collective Identity and Activism. In Morris, A. D. and Mueller, C. M., editors, Frontiers in Social Movement Theory, pages 156-173. Yale University Press, New Haven and London.

Frohlich, N. and Oppenheimer, J. A. (1970). I Get by with a Little Help from my Friends. World Politics, 23(1):105-120.

Ghosh, R. A., Glott, R., Krieger, B., and Robles, G. (2002). Free/Libre and Open Source Software: Survey and Study. Technical report, International Institute of Infonomics, University of Maastricht and Berlecon Research GmbH.

Granovetter, M. S. (1973). The Strength of Weak Ties. The American Journal of Sociology, 78(6):1360-1380.

Granovetter, M. S. (1983). The Strength of Weak Ties: A Network Theory revisited. Sociological Theory, 1:201-233.

Grieser, T. (2005). Market Analysis: Worldwide Distributed Performance and Availability Management Software 2005-2009 Forecast Summary and 2004 Vendor Shares. IDC No. 33752, IDC.

Hardin, R. (1982). Collective Action. John Hopkins Press, Baltimore, MD.

Hertel, G., Nieder, S., and Herrmann, S. (2003). Motivation of Software Developers in Open Source projects: an Internet-based Survey of Contributors to the Linux Kernel. Research Policy, 32(7):1159-1177. 
Houghton, J. W. (2004). Digital Delivery of Business Services. DSTI/ICCP/IE(2003)2/FINAL, OECD.

Jack, S. L. (2005). The Role, Use and Activation of Strong and Weak Network Ties: A Qualitative Analysis. Journal of Management Studies, 42(6):1233-1259.

Jeppesen, L. B. and Frederiksen, L. (2006). Why Do Users Contribute to Firm-Hosted User Communities? The Case of Computer-Controlled Music Instruments. Organization Science, 17(1):45-63.

Jeppesen, L. B. and Molin, M. J. (2003). Consumers as Co-developers: Learning and Innovation Outside the Firm. Technology Analysis \& Strategic Management, 15(3):363-383.

Lakhani, K. R. and von Hippel, E. (2003). How Open Source Software works: "Free" User-to-User Assistance. Research Policy, 32(6):923-943.

Lakhani, K. R. and Wolf, R. G. (2005). Why Hackers Do What They Do: Understanding Motivation and Effort in Free/Open Source Software Projects. In Feller, J., Fitzgerald, B., Hissam, S., and Lakhani, K. R., editors, Perspectives on Free and Open Source Software. The MIT Press, Cambridge-London.

Lau, D. C. and Murninghan, J. K. (1998). Demographic Diversity and Faultlines: The Compositional Dynamics of Organizational Groups. Academy of Management Review, 23(2):325-340.

Lazar, J. and Preece, J. (2002). Social considerations in online communities : usability, sociability, and success factors. In Oostendorp, H. V., editor, Cognition in a Digital World, pages 127-151. Lawrence Erlbaum Associates Inc. Publishers, Mahwah: NJ.

Lerner, J. and Tirole, J. (2001). The Open Source Movement: Key Research Questions. European Economic Review, 45(4-6):819-826.

Lerner, J. and Tirole, J. (2002). Some Simple Economics of Open Source. The Journal of Industrial Economics, 50(2):197-243.

Mayrhofer, P. (2005). Does Commercial Interest Hinder Innovation in User Communities? - A Theoretical and Empirical Study. unpublished Masters thesis, University of Munich.

McDermott, R. (2002). Measuring the impact of communities - how to draw meaning from measures of communities of practice. Knowledge Management Review, 5(2):26-29.

Melton, A. W. and von Lackum, W. J. (1941). Retroactive and proactive inhibition in retention: Evidence for a two-factor theory of retroactive inhibition. American Journal of Psychology, 54:157-173. 
Mockus, A., Fielding, R. T., and Herbsleb, J. (2000). A Case Study of Open Source Software Development: The Apache Server. In Proceedings of of the 22nd International Conference on Software Engineering. ACM Press.

Oliver, P. (1980). Rewards and Punishments as Selective Incentives for Collective Action: Theoretical Investigations. American Journal of Sociology, 85(6):1356-1375.

Olson, M. (1965). The Logic of Collective Action - Public Goods and the Theory of Groups. Harvard University Press, Cambridge-London.

Ostrom, E. (1998). A Behavioral Approach to the Rational Choice Theory of Collective Action. American Political Science Review, 92(1):1-22.

Ostrom, E., Gardner, R., and Walker, J. (1994). Rules, Games, and Common-Pool Resources. University of Michigan Press, Ann Arbor.

Polyani, M. (1958). Personal Knowledge: Towards a Post-critical Philosophy. Routledge \& Kegen Paul, London.

Polyani, M. (1966). The Tacit Dimension. Doubleday and Co., Garden City, N.Y.

Prahalad, C. K. and Ramaswamy, V. (2000). Co-opting Customer Competence. Harvard Business Review, 78(1):79-87.

Prügl, R. and Schreier, M. (2006). Learning from leading-edge customers at The Sims: Opening up the innovation process using toolkits. $R \& D$ Management, 36(3):237-250.

Raymond, E. S. (1999). The Cathedral and the Bazaar. In The Cathedral and the Bazaar: Musings on Linux and Open Source by an Accidental Revolutionary, pages 27-78. O'Reily \& Associates, Inc., Cambridge.

Rogers, W. H. (1993). Regression standard errors in clustered samples. Technical report, Stata Technical Bulletin 13: 19-23. Reprinted in Stata Technical Bulletin Reprints, vol. 3, 88-94.

Ryan, R. M. and Deci, E. L. (2000). Intrinsic and Extrinsic Motivations: Classic Definitions and New Directions. Contemporary Educational Psychology, 25(1):54-67.

Sally, D. (1995). Conservation and Cooperation in Social Dilemmas. A Meta-Analysis of Experiments from 1958 to 1992. Rationality and Society, 7:58-92.

Shah, S. K. (2005). Open Beyond Software. In Cooper, D., DiBona, C., and Stone, M., editors, Open Sources 2.0: The Continuing Evolution. O'Reilly Media: Sebastopol, CA. 
Swanson, G. E. (1992). Doing Things Together: Some Basic Forms of Agency and Structure in Collective Action and Some Explanations. Social Psychology Quarterly, 55(2):94-117.

Taylor, M. and Singleton, S. (1993). The Communal Resource: Transaction Costs and the Solution of Collective Action Problems. Politics and Society, 21(2):195-214.

Tobin, J. (1956). Estimation of Relationships for Limited Dependent Variables. Econometrica, 26:24-36.

Torvalds, L. and Diamond, D. (2001). Just for Fun: The Story of an Accidental Revolutionary. HarperBusiness, New York.

Urban, G. L. and von Hippel, E. (1988). Lead user analyses for the development of new industrial products. Management Science, 34(5):569-582.

von Hippel, E. (2001). Innovation by user communities: Learning from open-source software. MIT Sloan Management Review, 42:82-86.

von Hippel, E. (2002). Horizontal innovation networks - by and for users. Working Paper 4366-02, MIT Sloan School of Management.

von Hippel, E. and von Krogh, G. (2003). Open Source Software and the "Private-Collective" Innovation Model: Issues for Organization Science. Organization Science, 14(2):209-223.

von Krogh, G. (1998). Care in Knowledge Creation. California Management Review, 40(3).

von Krogh, G., Haefliger, S., and Spaeth, S. (2003a). Collective Action and Communal Resources in Open Source Software Development: The Case of Freenet. unpublished working paper, Institute of Manangement, University of St. Gallen, Switzerland.

von Krogh, G., Lakhani, K., and Spaeth, S. (2003b). Community Joining, and Specialization in Open Source Software Innovation. Research Policy, 32(7):1217-1241.

Ward, L. B. (1937). Reminiscence and rote learning. Psychological Monographs, 49(4).

Wasserman, S. and Faust, K. (1994). Social Network Analysis: Methods and Applications. Cambridge University Press, Cambridge-New York-Melbourne.

Weber, S. (2004). The Success of Open Source. Harvard University Press, Cambridge-London.

Wenger, E. (2004). Knowledge Management as a doughnut: Shaping your knowledge strategy through communities of practice. Ivey Business Journal, 68(3):1-8. 
Williams, R. L. (2000). A Note on Robust Variance Estimation for Cluster-Correlated Data. Technical report, Biometrics Volume 56 Page 645.

Wooldridge, J. M. (2002). Econometrics Analysis of Cross Section and Panel Data. MIT Press: Cambridge-London.

Zeitlyn, D. (2003). Gift Economies in the Development of Open Source Software: Anthropological Reflections. Research Policy, 32(7):1287-1291.

Zucker, L. G. and Darby, M. R. (1996). Star Scientists and Institutional Transformation: Patterns of Invention and Innovation in the Formation of the Biotechnology Industry. Proceedings of the National Academy of Sciences, 93(23):12709-12716. 


\section{A Appendix}

Due to the wide variety of software products CA offers, it is not possible to provide detailed descriptions of each product. However, a brief introduction of CA's six main product categories will be presented in the following ${ }^{32}$.

- Operations Management: CA's Unicenter operations management solutions provide network, systems, database, applications, and web infrastructure management. These solutions map IT infrastructure to business processes and automatically detect, diagnose, repair and recover complex problems across the entire technology stack supporting critical business applications and services. Additionally, they also streamline the delivery of critical software updates and patches to internal customers; as well as automate and optimize jobs and business workloads.

- Storage Management: BrightStor intelligent storage management solutions enable the proactive simplification, secure management and protection of information and storage assets.

- Data Management and Application Development: CA's Advantage $e^{33}$ software provide solutions for application development and generation, application code validation, testing and fault management; enterprise database and data management; and data access, transformation and transportation, for both the mainframe and distributed environment.

- Security Management: The eTrust security management solutions manages companies' entire security environment by integrating three key components - identity and access management, threat management and security information management.

- Life Cycle Management: AllFusion life cycle management solutions empowers companies to centrally store, administer, secure, version, and track the entire software inventory of their enterprise. These solutions provide for the integration and synchronisation of all software running on any platform, including mainframe, distributed, and web applications.

- Service Management: The CleverPath solutions from CA enable businesses to centralise information access; refine, analyse and sort data; create and distribute informative reports; and develop decision support systems that mirror their business processes.

\footnotetext{
${ }^{32}$ See CA's home page for detailed information on each product category (http://www3.ca.com/products/, accessed on 19th December 2004 and 22nd August 2005).

${ }^{33} \mathrm{CA}$ has for marketing purposes, recently shifted a number of application development software from the Advantage suite to the Allfusion suite.
} 